\title{
Towards Evaluating Fault Coverage of Protocol Test Sequences*
}

\author{
Jinsong Zhu and Samuel T. Chanson
}

Department of Computer Science, University of British Columbia

Vancouver, B.C., Canada V6T 1 Z4

In this paper, we investigate the quality of a given protocol test sequence in detecting faulty implementations of a specification. The proposed method differs from the conventional simulation method in that it is not necessary to consider various forms of fault combinations and is guaranteed to identify any faulty machines. Preprocessing and backjumping techniques are used to reduce the computational complexity of the method. We have constructed a tool based on the model and used it in assessing several test sequence generation techniques. Our experience shows that the method is both effective and efficient for even real life protocols.

Keyword Codes: C.2.2; C.2.m

Keywords: Protocol Specification and Testing; Fault Models; Fault Coverage

\section{Introduction}

Test sequence generation for communication protocols has been an active research area. However, the evaluation of the fault coverage for a given test sequence remains an open problem. Only a few methods such as the W method [5] and the DS method [9] have been proven to generate test sequences that can uniquely identify a finite state machine (FSM) under test. However, the W sequence is typically a lot longer than those generated using other methods, and many of the finite state machines simply do not possess a DS sequence [10]. On the other hand, other methods with better applicability and produces shorter sequence length, such as the UIO method [12] and a group of UIO-based optimization methods $[1,4,11,13]$, cannot in general guarantee to produce a checking experiment for a particular machine $[17,19]$. The reason is that these methods are all based on a correct specification but unlike the W and DS methods, cannot guarantee an equivalence mapping between the specification and a machine that accepts the sequence. Therefore, in principle one needs to check the coverage of a given test sequence generated by these methods.

In $[6,15]$, a simulation method was proposed to estimate the fault coverage of a given test sequence. Based on a classification of faults, it randomly generates faulty machines to see if they can be defeated by the test sequence. The number of machines that cannot be defeated is used as a measure of fault coverage. A limitation of the method is that because of the complicated fault behaviors, it is difficult, if not impossible, to come up

*This work was partially supported by a grant from the Canadian Institute for Telecommunications Research under the NCE program of the government of Canada. 
with a complete fault classification. The sampling of a small fraction of all cases also means the results must be interpreted with some degree of uncertainty.

in [18], a method was proposed for test sequence generation. The idea was to generate machines that accept ${ }^{2}$ an initial test sequence, and then incrementally add new subsequences to distinguish the machines until only one machine (the specification machine) is left. In this paper we extend this idea to coverage evaluation. A test sequence to be evaluated is used as the initial sequence. All machines that accept the sequence are referred to as indistinguishable. The number of indistinguishable machines, say $k$, with regard to a test sequence is used as a measure of its fault coverage. Obviously, the smaller the value of $k$, the fewer the machines that cannot be detected by the test sequence as faulty. This technique will uncover all faults and eliminates the need to explicitly consider various fault combinations.

We also propose a new method that fully takes advantage of the properties of the deterministic Finite State Machine (FSM) to overcome the problem of high computational complexity in [18]. By studying these properties, we found that techniques such as domain reduction and backjumping (see Section 3) can effectively prune the search space and substantially improve performance. Even a large real life protocol like NBS TP4 can be efficiently handled (see Table 2). This was a significant proof of the effectiveness of our method.

The rest of the paper is organized as follows. Section 2 contains a description of the underlying model and the properties of the deterministic FSM which forms the foundation of our coverage evaluation methodology detailed in Section 3. Section 4 shows the effectiveness of the method for a real life ISDN (Integrated Services Digital Network) protocol and Section 5 gives some empirical performance of the algorithms. Finally, Section 6 concludes the paper.

\section{The Underlying Model}

The underlying model in our study is a deterministic FSM. It is used in modeling the control part of a protocol (other techniques have been proposed for testing the data part, see for example [16, 3]). A deterministic FSM can be represented by a quintuple $M=\langle Q, X, Y, \delta, \lambda>$, where $Q, X, Y$ are the internal states, input alphabet and output alphabet respectively. $\delta$ (the next state function) is a mapping of $Q \times X$ into $Q$, and $\lambda$ (the output function) is a mapping of $Q \times X$ into $Y$. The functions $\delta$ and $\lambda$ can be extended for an input sequence $\sigma=x_{1} x_{2} \ldots x_{k}$ as usual: $\delta\left(q_{1}, \sigma\right)$ is the final state after $\sigma$ is applied to state $q_{1}$, and $\lambda\left(q_{1}, \sigma\right)$ denotes the corresponding output sequence. That is, $\lambda\left(q_{1}, \sigma\right)=y_{1} y_{2} \ldots y_{k}$ where $y_{i}=\lambda\left(q_{i}, x_{i}\right)$ and $q_{i+1}=\delta\left(q_{i}, x_{i}\right)$ for $i=1, \ldots, k$, and $\delta\left(q_{1}, \sigma\right)=q_{k+1}$.

If a state of an FSM is designated as the initial state (denoted as $q_{0}$ ), the FSM is said to be initialized. For simplicity, we will use initialized FSMs in the examples throughout this paper. The method is also applicable to uninitialized FSMs as long as a test sequence for each possible initial state is available.

An FSM is deterministic if any input symbol fed to the FSM causes a unique transition,

\footnotetext{
2 "Accept" here means the machine transfers to a valid state after the input sequence is applied, and generates the exact output sequence as specified in the test sequence.
} 
i.e.,

$$
\forall q_{i}, q_{j}, q_{k} \in Q \forall x \in X\left(\delta\left(q_{i}, x\right)=q_{j} \wedge \delta\left(q_{i}, x\right)=q_{k} \Leftrightarrow q_{j}=q_{k}\right) .
$$

It can be derived from $\mathrm{P} 1$ that if two states produce different outputs for the same input, then the two states must be distinct, i.e.,

$$
\forall q_{i}, q_{j} \in Q \forall x \in X\left(\lambda\left(q_{i}, x\right) \neq \lambda\left(q_{j}, x\right) \Rightarrow q_{i} \neq q_{j}\right) .
$$

A corollary of P2 is that if two states produce different output sequences under the same input sequence, then the two states must be different. Let $X^{*}$ denote the set of finite-length input sequences, then

$$
\forall q_{i}, q_{j} \in Q \forall \sigma \in X^{*}\left(\lambda\left(q_{i}, \sigma\right) \neq \lambda\left(q_{j}, \sigma\right) \Rightarrow q_{i} \neq q_{j}\right) .
$$

For a given input output sequence, if we do not limit the number of states and the input alphabet, there would be an infinite number of automata that "implement" the sequence and the number of indistinguishable FSMs would be infinite. We therefore only consider indistinguishable FSMs which have the same input alphabet as the specification FSM and which contain at most $i$ states more than that of the specification, where $i$ is a small integer. We will study the cases $i=0$ and $i=1$ as examples. For $i=0$, conformance checking can be done using the usual machine equivalence algorithm $[6,15]$ (note that V-equivalence [5] is used in this study as the equivalence relation between two automata), since in this case, conformance means equivalence, and vice versa. For $i>0$, the generated FSM is minimized before being checked. Other faults considered in this study are the usual ones, i.e., output faults, transfer faults, and their combinations.

To avoid equivalent states, the FSM is assumed to be minimal. This is justifiable because we can always reduce an FSM to its minimal form [8]. Furthermore, testing can only determine an implementation's conformance up to the level of equivalence. The machine should also be strongly connected and fully specified. Strong connectivity ensures each state can be reached from any other state ${ }^{3}$ For partially specified machines, we use the completeness assumption that the machine will remain in the present state without producing any output (or null output) for any unspecified input.

In the following sections, we shall also use the graph representation $G$ of an FSM. $G$ is a directed graph $(V, E)$, where the vertex set $V$ denotes the set of states, and the edge set $E$ represents the transitions, i.e., $V=\left\{q_{0}, \ldots, q_{n-1}\right\}, E=\left\{\left(q_{i}, q_{j}\right) \mid i, j \leq n-1\right.$ and there is a transition from $q_{i}$ to $\left.q_{j}\right\}$. An edge from $q_{i}$ to $q_{j}$, which receives input $a_{k}$ and produces output $o_{l}$, is labeled by $\left(q_{i}, q_{j} ; L\right)$ where $L \equiv a_{k} / o_{l}$, the input part of $L$ is denoted $L^{(i)} \equiv a_{k}$, and the output part of $L$ is $L^{(o)} \equiv o_{l}$.

\section{Coverage Evaluation Methodology}

As mentioned in Section 1, the coverage of a test sequence is measured by the number of indistinguishable machines which have the same input alphabet as the specification FSM and with an upper bound on the number of states. The problem thus becomes how to generate these indistinguishable machines under the given conditions. This problem

\footnotetext{
${ }^{3}$ The strong connectivity assumption can be replaced by a weaker one where every state can be reached from the initial state.
} 
can be viewed as the reverse procedure of test sequence generation. The test sequence can be thought of as an unfolding of the FSM. The idea is to "collapse" the test sequence back to one or more FSMs which may or may not conform to the original specification. A FSM so constructed is called a solution with the given input alphabet and number of states. If only one solution is obtained, then it must conform to the specification and the number of indistinguishable FSM is zero. In other words, the coverage is $100 \%$.

A FSM is said to have a reset capability (or resettable) if a special input signal ri always correctly sets the machine to its initial state $q_{0}$ from any state. Otherwise, it is called a resetless machine. In the following procedure we will study resettable machines only. For resetless machines, the entire test sequence can be considered as a single subsequence and is therefore a special case of the first situation.

For a resettable machine, a test sequence consists of test subsequences which start from $q_{0}$. Based on the properties of the deterministic FSM, we can construct a test tree with these subsequences as follows. The root of the tree is $q_{0}$. Each node corresponds to a state in the specification. The edges from a node to its children represent outgoing transitions from the corresponding state for each input symbol. Input symbols at every node are arranged in a fixed order to avoid isomorphic trees. The depth of the tree is the length of the longest subsequence. For a resetless machine, the test tree degenerates to a simple path. Figure 1 shows a sample FSM (taken from [17]), its UIO test sequence, and the corresponding test tree. Clearly, this tree can be constructed efficiently (in polynomial time) from the test sequence. The properties P1 and P2 guarantee a unique tree for a given test sequence.

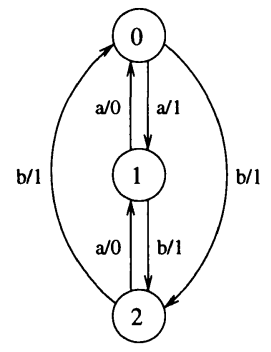

UIO Test Sequence:

ri/- $\mathrm{a} / 1 \mathrm{a} / 0 \mathrm{a} / 1$

$\mathrm{ri} /-\mathrm{b} / 1 \mathrm{~b} / 1 \mathrm{a} / 1$

ri/- $a / 1 b / 1 b / 1 a / 1$

$\mathrm{ri} /-\mathrm{a} / 1 \mathrm{~b} / 1 \mathrm{a} / 0 \mathrm{a} / 0 \mathrm{a} / 1$

(UIO for each state:

$0: a / 1 ; 1: a / 0 a / 1 ; 2: b / 1 a / 1)$

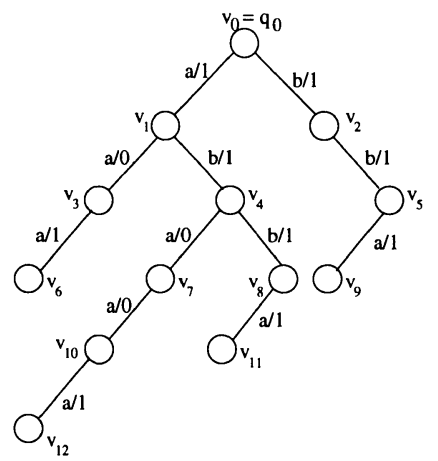

Figure 1. Test tree corresponding to the UIO test sequence

Now we assign each node a variable $v_{i}$, with $i$ numbered according to the node's breadth-first traversal order in the tree (see Figure 1). $v_{0}$ is always assigned to the root $q_{0}$. Where there is no ambiguity, we shall use the term node and variable interchangeably. The number of variables $l$ (other than $v_{0}$ ) can be determined by a breadth-first traversal of the tree. For example, in Figure $1, l=12$. Initially, each variable can represent any state. Suppose the specification FSM has $n$ states: $Q=\left\{q_{0}, q_{1}, \ldots, q_{n-1}\right\}$, then the domain of each variable is $Q$. A consistent instantiation of all variables constitutes a so- 
lution FSM, which is either the specification FSM or an indistinguishable machine. If we search all variables to generate the solutions as was done in [18], the computational complexity would be $O\left(n^{l}\right)$. The following techniques are used to reduce the computational complexity.

(1) Preprocessing: Although the domain of each variable is $Q$, the deterministic property will often restrict the values that a variable can assume. For example, according to P2, a variable cannot take on the value (i.e., a state) of a previous variable unless they are equal. In the extreme case, each variable may only have one value, representing the given FSM. We reduce the domains of the variables in the breadth-first order. For each variable $v_{i}$, we can obtain a set of variables, called unequal variables of $v_{i}$, whose indices are smaller than $i$ and whose values are different from that of $v_{i}$. Variables that can only have one value constitute the unique set. Initially, the unique set contains $v_{0}$ only. The uniqueness of a variable $v_{i}$ can be determined by examining its set of unequal variables. If this set contains the current unique set, then $v_{i}$ must itself be a uniquely determined variable and is added to the unique set. To prevent isomorphic solutions, the unique state $q_{j}$ assigned to $v_{i}$ is chosen such that $j$ is the smallest index not yet assigned. For example, if $v_{i}$ is the first one that differs from $v_{0}$, then it is assigned $q_{1}$. The first variable that is distinct from $v_{0}$ and $v_{i}$ is then assigned the next unassigned state, $q_{2}$. A variable that is not in the unique set but has some unique states in its set of unequal variables can have these unique states removed from its domain. This is because it is not possible for the variable to assume any of these values. This procedure is performed until all variables have been processed. The results are a reduced domain and a set of unequal variables for each variable. The preprocessing phase often prunes the search space significantly and saves considerable time in the subsequent searches. Furthermore, for any variable $v_{j}$ which is not uniquely determined, its set of unequal variables can help to reduce the search space dynamically, since it will not be necessary to assign $v_{j}$ a value which has already been assigned to any of its unequal variables.

(2) Backjumping: During searching, when a variable cannot be assigned any value which is consistent with the previous assignments (a dead-end situation), we can jump back to the variable which causes the inconsistency rather than backtracking one step at a time as is usually done. This idea is widely used in solving search problems [7]. The point is to go back to the source of failure as far as possible. In our problem, when a variable is instantiated, it may be forced to take a value in one of two ways. First, it may only take a single value if its domain size is one. Second, the assignment of a previous variable which has the same input symbol may force it to assume the same value in order to be consistent with the properties of the deterministic FSM. Such value-forced variables cannot be the source of failures, so when a dead-end is encountered, they need not be reconsidered in selecting candidates. This situation will occur very frequently when the test sequence contains many identical transitions.

The algorithms for preprocessing and backjumping search are given below.

\section{Algorithm-PREP: Domain reduction of node variables}

Input: Node variables $v_{i}, i=1, . ., l$

Output: Reduced domain $D_{i}$ for each $v_{i}$ and its unequal variables $N E Q_{i}$. 
Step 1: Generate unequal variables:

Initially, the set of unequal variables for each $v_{i}, N E Q_{i}=\emptyset$.

for every $v_{i}(1 \leq i \leq l)$ do

for every $v_{j}$ with $j<i$ do

if $\left(v_{i} \neq v_{j}\right)$ then add $v_{j}$ to $N E Q_{i}$.

Step 2: Reduce domains of variables:

Initially, the set of uniquely determined variables $U=\left\{v_{0}\right\}$, state set $S=\left\{q_{1}, \ldots, q_{n-1}\right\}$, and the domain for $v_{i}(i=1, \ldots, l)$ is $D_{i}=S \cup\left\{q_{0}\right\}$.

for every $v_{i}(1 \leq i \leq l)$ do

if $U \subset N E Q_{i}$ then begin

add $v_{i}$ to $U$;

$D_{i} \leftarrow\left\{q_{k}\right\}, k=$ the smallest subscript in $S$;

$S \leftarrow S-\left\{q_{k}\right\}$

for all $v_{j} \in N E Q_{i}$ do

end else

$D_{j} \leftarrow D_{j}-\left\{q_{k}\right\}$

for all $v_{j} \in N E Q_{i}$ do

if $v_{j} \in U$ then

$D_{i} \leftarrow D_{i}-D_{j} ;$

In Step 1, the set of unequal variables for each variable is generated. The if statement is executed $l(l-1) / 2$ times. The evaluation of the condition $v_{i} \neq v_{j}$ is based on property P3. Since input sequences starting from a node constitute a subtree rooted at that node, the evaluation can be performed by comparing two subtrees rooted at $v_{i}$ and $v_{j}$ respectively. The comparison can be done by means of a breadth-first search algorithm for the subtree. When comparing two nodes, property P2 is used. When a pair of distinct nodes is found, the two subtrees are distinct (P3). However, when subtrees are incomplete, the node with an absent edge is not considered distinct from its corresponding node where the edge is present. For example, in Figure 1, although $v_{2}$ has no edge corresponding to $a$, we cannot conclude $v_{2}$ must be different from $v_{0}$. Similarly, $v_{5}$ may possibly be equal to $v_{2}$. The time complexity of tree comparison is at most $O(l)$. Hence, the complexity of Step 1 is $O\left(l^{3}\right)$.

The domains are reduced in Step 2 using the $N E Q$ sets. If $N E Q_{i}$ contains the current uniquely determined variables, $v_{i}$ itself becomes a member of $U$. For each member $v_{j}$ of $U$, the domains of $v_{j}$ 's $N E Q$ variables can be reduced by removing $v_{j}$ 's corresponding value. The idea is that if a variable is not equal to a uniquely determined variable, it cannot assume the unique value of that variable. The complexity of this step is $O\left(l^{2}\right)$. Thus, the overall complexity of Algorithm-PREP is $O\left(l^{3}\right)$. Note that the test tree has to be constructed from the test sequence before using this algorithm.

Algorithm-SEARCH: Backjumping search to find solutions

Input: Node variables with reduced domains and the original specification

Output: The set of indistinguishable FSMs 
Initially, the index of variables $i=1$. A stack is used to store intermediate steps.

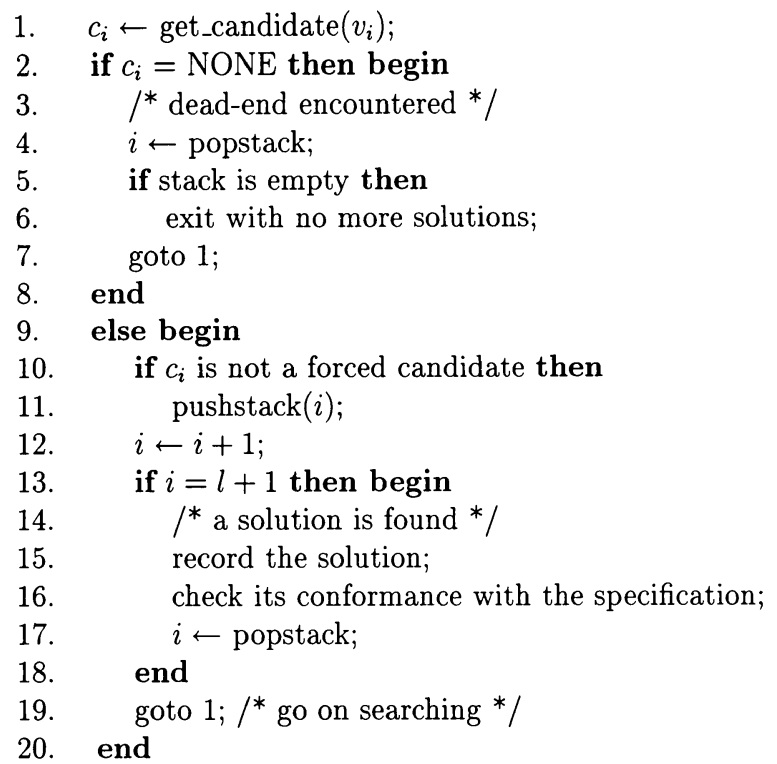

The procedure get_candidate in line 1 selects a consistent value for $v_{i}$ from its domain (we shall say that $v_{i}$ is instantiated). The reduced domain and the set $N E Q_{i}$ both help reduce the number of candidates. During the instantiation process, it builds up a partial solution FSM with the values of variables up to $v_{i}$, one by one. To determine a consistent value of $v_{i}$, let $v_{p}$ be $v_{i}$ 's parent node in the test tree, and the I/O label from $v_{p}$ to $v_{i}$ be $x / y$. Suppose $v_{p}$ has the value $q_{p}$ which is a state in the partial FSM. If $q_{p}$ has no outgoing transition labeled $x / y$, then we get a value for $v_{i}$ which is the next state (say $q_{t}$ ) in its domain, and a new transition from $q_{p}$ to $q_{t}$ is added to the partial FSM. Otherwise, $v_{i}$ is forced to be $\delta\left(q_{p}, i\right)$ in the FSM, because for a label $x / y$ from a state $q_{p}$ we can have at most one transition (refer to P1). The partial FSM is constructed incrementally until all variables are assigned consistent values, at which time the FSM becomes a final solution. To avoid isomorphic solutions, when instantiating $v_{i}$, only one value out of a set of equivalent candidates is selected. The candidates are equivalent in the sense that they represent equivalent states in the current partial FSM. If one of the equivalent candidates fails, the others will fail too. This fact is used to reduce the domains to be searched dynamically. Get_candidate returns NONE when no consistent value of $v_{i}$ can be found. This is the dead-end situation so backjumping is invoked. Since a forced variable cannot be the source of a failure as mentioned before, line 11 only pushes an unforced variable onto the stack for subsequent backtracking. In line $13, i=l+1$ means all variables have been successfully instantiated, and a solution is found. Line 16 checks the solution's conformance with the specification using Dahbura and Sabnani's algorithm [6]. A non-conforming solution represents an indistinguishable FSM. 


\section{An Example}

We shall use the FSM in Figure 1 to illustrate the above algorithms, Initially, $D_{0}=$ $\left\{q_{0}\right\}, D_{i}=\left\{q_{0}, q_{1}, q_{2}\right\}$ for $i=1, \ldots, 12$. From the tree, it can be seen that $v_{1}$ must be different from $v_{0}$ as their outputs to $a$ are different. At this point, only $v_{0}$ is in the unique set $U$, so $v_{1}$ is also a uniquely determined variable. $v_{1}$ is then assigned $q_{1}$, the next value in the state set. Next, $v_{2}$ is found to be distinct from $v_{1}$ because of their different outputs for $b a$. Therefore $v_{1}$ 's value $q_{1}$ is removed from $v_{2}$ 's domain. After all variables have been processed, their domains are found to be:

$$
\begin{aligned}
& D_{1}=\left\{q_{1}\right\}, D_{2}=\left\{q_{0}, q_{2}\right\}, D_{3}=\left\{q_{0}\right\}, D_{4}=\left\{q_{2}\right\}, \\
& D_{5}=\left\{q_{0}\right\}, D_{6}=\left\{q_{0}, q_{1}, q_{2}\right\}, D_{7}=\left\{q_{1}\right\}, D_{8}=\left\{q_{0}\right\}, \\
& D_{9}=\left\{q_{0}, q_{1}, q_{2}\right\}, D_{10}=\left\{q_{0}\right\}, D_{11}=\left\{q_{0}, q_{1}, q_{2}\right\}, D_{12}=\left\{q_{0}, q_{1}, q_{2}\right\} .
\end{aligned}
$$

Note that the domains for most nodes have reduced, some of which have only one candidate. Those unreduced domains are all for leaf nodes since they have no outgoing edges (we'll see that this does not affect the search process). Now Algorithm-SEARCH is used to search for solutions. For the first five variables, only $v_{2}$ has more than one choice. We first choose $q_{0}$, which is consistent with $v_{1}=q_{1}$. The remaining variables are forced to take the values $q_{1}, q_{1}, q_{0}, q_{1}, q_{0}, q_{1}$, and $q_{1}$ respectively. Thus, we obtain our first solution:

$$
\begin{aligned}
& v_{0}=q_{0}, v_{1}=q_{1}, v_{2}=q_{0}, v_{3}=q_{0}, v_{4}=q_{2}, v_{5}=q_{0}, v_{6}=q_{1}, \\
& v_{7}=q_{1}, v_{8}=q_{0}, v_{9}=q_{1}, v_{10}=q_{0}, v_{11}=q_{1}, v_{12}=q_{1} .
\end{aligned}
$$

Since all variables after $v_{2}$ were forced, the algorithm goes directly back to $v_{2}$ to find the next solution. The next candidate for $v_{2}$ is $q_{2}$, which is also consistent with $v_{1}=q_{1}$. The other variables are again forced to the same values, producing another solution:

$$
\begin{aligned}
& v_{0}=q_{0}, v_{1}=q_{1}, v_{2}=q_{2}, v_{3}=q_{0}, v_{4}=q_{2}, v_{5}=q_{0}, v_{6}=q_{1}, \\
& v_{7}=q_{1}, v_{8}=q_{0}, v_{9}=q_{1}, v_{10}=q_{0}, v_{11}=q_{1}, v_{12}=q_{1} .
\end{aligned}
$$

It can be seen that the variables cannot take on any other value and so the algorithm terminates with two solutions. Figure 2 shows the two corresponding FSMs, with the first one an indistinguishable FSM, and the second one conforming to the specification. The

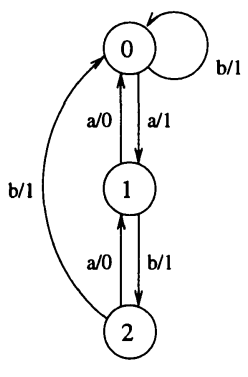

(a) Solution 1: indistinguishable FSM

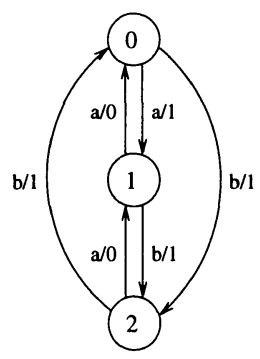

(b) Solution 2: conforming FSM

Figure 2. The two solutions for the UIO test sequence given in Figure 1 
two solutions are both obtained backtrack-free, which means only $O(l)$ time is needed to get a solution. This is the best result we can hope for. We also observed that although domains for the leaf nodes cannot be reduced, they are usually forced to some values. For example, $v_{6}, v_{9}, v_{11}$, and $v_{12}$ were all forced variables.

\section{Q.931 Network Side Protocol as an Example}

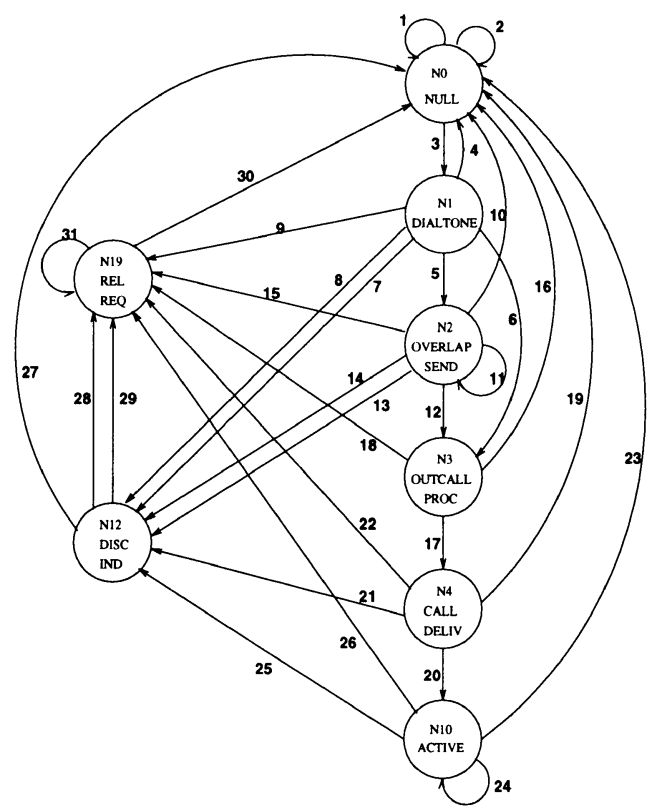

1. Ui_1 1 eotup_badnil_1trelcom

2. Ui_17reivi_itrelcom

3. UI_1?setup/UI_1leotupack

4,10. UI_1?relvUI_1?rolcom

5. UI_1 info/vi_ilinfo

6. UI_1 ?into_last/UI_1linfo.UI_1tcallproc

7,13. T3027timeourVII_1prog.UI_1ldisc

8,14. UI_1?into_badVII_1lprog.UI_1ldisc

9. UI__1?disc/UI_1trel

11. UI_1 1 info/null

12. UI_1?info_last/UI_1lcallproc

15. UI_17disc/UI_1trol

16.UI_1?rol/UI_1lroicom

17. NI_2?netalertUI_1talert

18. UI_1?disc/UI_1Irel

19. UI_1?re//UI_1!rolcom

20. NI_2?netconn/UI_1lconn

21. NI_2?netclear/NI_1ldisc

22. UI_12disc/Ni_1trel

23. UI_1?rel/II_1trelcom

24. UI_17connack/null

25. NI_2?netclear/NI_1Idisc

26. Ui_1?disc/UI_1Irel

27. UI_1?reVUI_1trelcom

28. UI_1?disc/UI_1Irel

29. T305?timeout/UI_1!rel

30. T308?timeout/UI_1!rel

31. UI_17disc/null

Figure 3. ISDN BRI network layer protocol (network side at the originating side)

The above example is too small to be practical. To show the effectiveness of the method to real life protocols, we take the example in [1], which is part of the ISDN Basic Rate Interface (BRI) D-channel signaling protocol. The state diagram of the protocol is shown in Figure 3 (taken from [1]). This protocol is defined in CCITT Recommendation Q.931 [2] (network layer) as a user-network interface. It describes the procedures for establishing, maintaining, and clearing basic circuit-switched voice connections. Only the network side of the Q.931 BRI for basic voice services at the originating end is considered here.

The notation used in Figure 3 is the same as in [1], i.e., the transition label $A ? m_{i} / B ! m_{j}$ is interpreated as receiving the input message $m_{i}$ from FSM A, and sending the output message $m_{j}$ to FSM B. Table 1 lists the UIO sequences for each state, and Table 3 gives all the test subsequences, with reset capability assumed. There are a total of 8 states, 14 inputs, 12 outputs, and 425 test steps (transitions). 
Table 1. UIO Sequences for the FSM in Figure 3

\begin{tabular}{|l|l|}
\hline State & \multicolumn{1}{|c|}{ UIO Sequence } \\
\hline N0 & UI_1?setup_bad/UI_1!relcom \\
N1 & UI_1?info/UI_1!info \\
N2 & UI_1?info/null \\
N3 & NI_2?netalert/UI_1!aler \\
N4 & NI_2?netconn/UI_1!conn \\
N10 & UI_1?connack/null \\
N12 & T305!timeout/UI_1!rel \\
N19 & UI_1?disc/null \\
\hline
\end{tabular}

A tool based on Section 3 has been implemented in C and runs on a Sun 4/30 workstation under Sun OS 4.1.1. It accepts as input a test sequence and a specification FSM and outputs all solutions found with the algorithms in Section 3. We applied the test sequence to the tool, and in less than a second, the unique solution was found backtrackfree, i.e., the coverage of the test sequence is $100 \%$. This result is listed in Table 2 (see next section), among other experimental results.

\section{Other Empirical Results}

To collect more empirical results for problems of practical size, we conducted more experiments with examples taken from the literature. The actual computational cost is problem dependent. However, even though the complexity of Algorithm-SEARCH is $O\left(n^{l}\right)$ in the worst case when there is no reduction of domain for all variables and no forced variables, the results show that the complexity is far less in practice.

In $[6,15]$, some examples were used for fault coverage evaluation using simulation. We applied our tool to the examples, and derived the same conclusions. The W, DS and UIO methods were evaluated in [15]. The conclusion was that they all had the same full coverage for strong conformance test. Nevertheless, since the number of all possible machines is $15^{10}\left(\approx 5.7 \cdot 10^{11}\right)$ for the sample machine $M$ (Fig.1 in [15]), and only $10^{6}$ randomly generated machines were examined, the conclusion needs to be verified. With our tool, we were able to confirm that the three test sequences given in the paper did have a unique conforming solution (see Table 2 for FSM 1) and thus have a $100 \%$ fault coverage. The example in [6] (FSM 2) for UIO method also has a unique solution. An encouraging observation is that all solutions were obtained backtrack-free.

To further evaluate our tool, we studied the $\mathrm{W}$ method with one extra state (denoted as $\mathrm{W}^{+1}$ in Table 2), using the same sample FSM 1 . The original example for the DS method in [9] (FSM 3) was also studied for both the DS and UIO methods, assuming it is resettable. Furthermore, two real protocols, the ISDN BRI network layer protocol [1] and a subset of the NBS Class 4 transport protocol (TP4) [14], were studied for the UIO and DS methods respectively The fault coverages for these examples were all found to be $100 \%$.

Table 2 summarizes the results of our experiments. In the table, $(n, m, p, L)$ is the number of states, inputs and outputs for the example, and the length of the test sequence, 
Table 2. Performance of our method on some examples

\begin{tabular}{|c|c|c|c|c|c|c|}
\hline \multirow{2}{*}{ Example } & $(n, m, p, L)$ & Method & $\begin{array}{c}\text { No. of } \\
\text { Solutions }\end{array}$ & $\begin{array}{c}\text { No. of } \\
\text { Backtrackings }\end{array}$ & $\begin{array}{c}\text { No. of } \\
\text { Forced Cases }\end{array}$ & Time $(s)$ \\
\hline \hline \multirow{3}{*}{ FSM 1 } & $(5,2,3,29)$ & UIO & 1 & $(0,0)$ & $(9,9)$ & 0.04 \\
\cline { 2 - 7 }$[15]$ & $(5,2,3,35)$ & DS & 1 & $(0,0)$ & $(12,12)$ & 0.04 \\
\cline { 2 - 7 } & $(5,2,3,58)$ & W & 1 & $(0,0)$ & $(18,18)$ & 0.05 \\
\cline { 2 - 7 } & $(5,2,3,130)$ & $\mathrm{W}^{+1}$ & 15 & $(0,60)$ & $(48,558)$ & 0.15 \\
\hline FSM 2[6] & $(7,4,4,111)$ & UIO & 1 & $(0,15)$ & $(38,256)$ & 0.13 \\
\hline FSM 3 & $(6,2,2,40)$ & UIO & 1 & $(8,59)$ & $(35,203)$ & 0.09 \\
\cline { 2 - 7 }$[9]$ & $(6,2,2,62)$ & DS & 1 & $(0,0)$ & $(25,25)$ & 0.07 \\
\hline ISDN BRI[1] & $(8,14,12,425)$ & UIO & 1 & $(0,0)$ & $(105,105)$ & 0.82 \\
\hline NBS TP4[14] & $(15,27,26,4131)$ & DS & 1 & $(105,488)$ & $(2874,2909)$ & 273.77 \\
\hline \hline
\end{tabular}

respectively. The solutions for the examples we tried are all unique and conform to the original specification (no indistinguishable solutions). Two numbers are recorded in the column for "No. of Backtrackings" and also the column for "No. of Forced Cases". The first one is the number when the first solution is found, and the second when the algorithm stops. Forced cases occur when a variable is forced to a value during instantiation. The larger the number of forced cases, the better the gains from backjumping. The column "Time" is the number of seconds of CPU time consumed by the tool, including input and output processing. The results showed that the strategy of preprocessing and backjumping is surprisingly effective. Even a sizable real protocol like NBS TP4 can be handled efficiently. Considering the small number of backtrackings (many of which are backtrack-free) and the worst case complexity of $n^{l}$, the savings are substantial.

\section{Conclusions}

We have examined the issue of fault coverage evaluation of a given test sequence for deterministic FSMs. The metric for fault coverage is the number of indistinguishable machines and a tool has been constructed based on this model. Preprocessing and backjumping techniques have been proposed which reduce the complexity of finding indistinguishable FSMs substantially. The tool has been used in evaluating the quality of some test sequence generation techniques, and some previous results were confirmed. Other applications such as an evaluation of a group of UIO-based optimization techniques are reported in [19]. Since each indistinguishable FSM is explicitly generated, the tool is also useful in analyzing how a faulty machine could have escaped detection.

\section{REFERENCES}

1. A.V. Aho, A.T. Dahbura, D. Lee, and M.U. Uyar. An optimization technique for protocol conformance test generation based on UIO sequences and rural Chinese postman tours. IEEE Transactions on Communications, 39(11), November 1991.

2. CCITT Red Book, vol.III. FASCICLE III.5 - Integrated Services Digital Network (ISDN) Recommendations (Study Group XVIII). 1984.

3. S.T. Chanson and J. Zhu. A unified approach to protocol test sequence generation. In Proc. IEEE INFOCOM, San Francisco, March 1993. 
4. M.S. Chen, Y. Choi, and A. Kershenbaum. Approaches utilizing segment overlap to minimize test sequences. In Proc. IFIP 10th Int. Symp. on Protocol Specification, Testing, and Verification, 1990.

5. T.S. Chow. Testing software design modeled by finite-state machines. IEEE Transactions on Software Engineering, May 1978.

6. A. Dahbura and K. Sabnani. An experience in estimating fault coverage of a protocol test. In Proc. IEEE INFOCOM, 1988.

7. R. Dechter. Enhancement schemes for constraint processing: backjumping, learning, and cutset decomposition. Artificial Intelligence, 41(3):273-312, 1990.

8. A. Gill. Introduction to the Theory of Finite State Machines. McGraw-Hill Book Company, Inc., 1962.

9. G. Gonenc. A method for the design of fault detection experiments. IEEE Transactions on Computer, June 1970.

10. Z. Kohavi. Switching and Finite Automata Theory. New York: McGraw Hill, 1978.

11. R.E. Miller and S. Paul. Generating minimal length test sequences for conformance testing of communication protocols. IEEE/ACM Trans. on Network, Februry 1993.

12. K. Sabnani and A. Dahbura. A protocol test generation procedure. Computer Networks and ISDN Systems, 15:285-297, 1988.

13. Y.-N Shen, F. Lombardi, and A.T. Dahbura. Protocol conformance testing using multiple UIO sequences. In Proc. IFIP 9th Int. Symp. on Protocol Specification, Testing, and Verification, 1989.

14. D.P. Sidhu and T.-K. Leung. Formal methods for protocol testing: A detailed study. Technical Report 86-23, Dept of Computer Science, Iowa State Univ., 1986.

15. D.P. Sidhu and T.-K. Leung. Formal methods for protocol testing: A detailed study. IEEE Transactions on Software Engineering, April 1989.

16. H. Ural and B. Yang. A test sequence selection method for protocol testing. IEEE Transactions on Communication, April 1991.

17. S.T. Vuong and W.Y.L Chan. The UIOv-method for protocol test sequence generation. In Proc. 2nd Int. Workshop on Protocol Testing System, October 1989.

18. S.T. Vuong and K.C. Ko. A novel approach to protocol test sequence generation. In Proc. GLOBECOM'90, December 1990.

19. J. Zhu and S.T. Chanson. Fault coverage evaluation of protocol test sequences. Technical Report 93-19, Dept of Computer Science, Univ of British Columbia, June 1993. 
Table 3. UIO-based Test Sequences for the FSM in Figure 3 (cont'd)

r/- UI_1? setup/UI_1!setupack UI_1?info_last/UI_1!info.UI_1!callproc UI_1?rel/UI_1!relcom

UI_1?setup_bad/UI_1!relcom

r/- UI_1?setup/UI_1!setupack UI_1?info_last/UI_1!info.UI_1!callproc UI_1?disc/UI_1!rel

UI_1?disc/null

r/- UI_1?setup/UI_1!setupack UI_1?info_last/UI_1!info.UI_1!callproc T302?timeout/NI_2? netalert/UI_1!alert

r/- UI_1? setup/UI_1!setupack UI_1?info_last/UI_1!info.UI_1!callproc NI_2?netclear/UI_1!disc T305?timeout/UI_1!rel

r/- UI_1? setup/UI_1!setupack UI_1?info_ast/UI_1!info.UI_1!callproc NI_2?netconn/NI_2?netalert/UI_1!alert

r/- UI_1? setup/UI_1!setupack UI_1?info_last/UI_1!info.UI_1!callproc UI_1?connack/NI_2?netalert/UI_1!alert

r/- UI_1?setup/UI_1!setupack UI_1?info_last/UI_1!info.UI_1!callproc T305?timeout/NI_2?netalert/UI_1!alert

r/- UI_1?setup/UI_1!setupack UI_1?info_last/UI_1!info.UI_1!callproc T308?timeout/NI_2?netalert/UI_1!alert

r/- UI_1? setup/UI_1!setupack UI_1?info_last/UI_1!info.UI_1!callproc NI_2? netalert/UI_1!alert UI_1?setup_bad/- NI_2?netconn/UI_1!conn

r/- UI_1? setup/UI_1!setupack UI_1?info_last/UI_1!info.UI_1!callproc NI_2?netalert/UI_1!alert UI_1?setup/- NI_2?netconn/UI_1!conn

r/- UI_1? setup/UI_1!setupack UI_1?info_ast/UI_1!info.UI_1!callproc NI_2? netalert/UI_1!alert UI_1?info/- NI_2? netconn/UI_1!conn

r/- UI_1? setup/UI_1!setupack UI_1?info_last/UI_1!info.UI_1!callproc NI_2?netalert/UI_1!alert UI_1?info_bad/- NI_2? netconn/UI_1!conn

r/- UI_1?setup/UI_1!setupack UI_1?info_last/UI_1!info.UI_1!callproc NI_2?netalert/UI_1!alert UI_1?info_last/- NI_2? netconn/UI_1!conn

r/- UI_1?setup/UI_1!setupack UI_1?info_last/UI_1!info.UI_1!callproc NI_2?netalert/UI_1!alert UI_1?rel/UI_1!relcom UI_1?setup_bad/UI_1!relcom

r/- UI_1? setup/UI_1!setupack UI_1?info_last/UI_1!info.UI_1!callproc NI_2?netalert/UI_1!alert UI_1?disc/UI_1!rel UI_1?disc/null

r/- UI_1?setup/UI_1!setupack UI_1?info_last/UI_1!info.UI_1!callproc NI_2?netalert/UI_1!alert T302?timeout/- NI_2? netconn/UI_1!conn

r/- UI_1? setup/UI_1!setupack UI_1?info_last/UI_1!info.UI_1!callproc NI_2?netalert/UI_1!alert NI_2? netclear/UI_1!disc T305?timeout/UI_1!rel

r/- UI_1?setup/UI_1!setupack UI_1?info_last/UI_1!info.UI_1!callproc NI_2?netalert/UI_1!alert NI_2?netalert/- NI_2?netconn/UI_1!conn

r/- UI_1? setup/UI_1!setupack UI_1?info_last/UI_1!info.UI_1!callproc NI_2?netalert/UI_1!alert UI_1?connack/- NI_2?netconn/UI_1!conn

r/- UI_1? setup/UI_1!setupack UI_1?info_last/UI_1!info.UI_1!callproc NI_2?netalert/UI_1!alert T305?timeout/- NI_2?netconn/UI_1!conn

r/- UI_1? setup/UI_1!setupack UI_1?info_last/UI_1!info.UI_1!callproc NI_2?netalert/UI_1!alert T308?timeout/- NI_2? netconn/UI_1!conn

r/- UI_1? setup/UI_1!setupack UI_1?info_last/UI_1!info.UI_1!callproc NI_2?netalert/UI_1!alert NI_2?netconn/UI_1!conn UI_1?setup_bad/- UI_1?connack/null

r/- UI_1? setup/UI_1!setupack UI_1?info_last/UI_1!info.UI_1!callproc NI_2? netalert/UI_1!alert NI_2?netconn/UI_1!conn UI_1?setup/- UI_1?connack/null

r/- UI_1? setup/UI_1!setupack UI_1?info_last/UI_1!info.UI_1!callproc NI_2?netalert/UI_1!alert NI_2?netconn/UI_1!conn UI_1?info/- UI_1?connack/null

r/- UI_1? setup/UI_1!setupack UI_1?info_last/UI_1!info.UI_1!callproc NI_2?netalert/UI_1!alert NI_2?netconn/UI_1!conn UI_1?info_bad/- UI_1?connack/null 
Table 3. UIO-based Test Sequences for the FSM in Figure 3 (cont'd)

r/- UI_1? setup/UI_1!setupack UI_1?info_last/UI_1!info.UI_1!callproc NI_2?netalert/UI_1!alert NI_2?netconn/UI_1!conn UI_1?info_last/- UI_1?connack/null

r/- UI_1?setup/UI_1!setupack UI_1?info_last/UI_1!info.UI_1!callproc NI_2?netalert/UI_1!alert NI_2? netconn/UI_1!conn UI_1?rel/UI_1!relcom UI_1?setup_bad/UI_1!relcom

r/- UI_1?setup/UI_1!setupack UI_1?info_last/UI_1!info.UI_1!callproc NI_2?netalert/UI_1!alert NI_2?netconn/UI_1!conn UI_1?disc/UI_1!rel UI_1?disc/null

r/- UI_1? setup/UI_1!setupack UI_1?info_last/UI_1!info.UI_1!callproc NI_2?netalert/UI_1!alert NI_2? netconn/UI_1!conn T302?timeout/- UI_1?connack/null

r/- UI_1?setup/UI_1!setupack UI_1?info_last/UI_1!info.UI_1!callproc NI_2?netalert/UI_1!alert NI_2?netconn/UI_1!conn NI_2?netclear/UI_1!disc T305?timeout/UI_1!rel

r/- UI_1?setup/UI_1!setupack UI_1?info_last/UI_1!info.UI_1!callproc NI_2?netalert/UI_1! alert NI_2?netconn/UI_1!conn NI_2? netalert/- UI_1?connack/null

r/- UI_1? setup/UI_1!setupack UI_1?info_last/UI_1!info.UI_1!callproc NI_2?netalert/UI_1!alert NI_2?netconn/UI_1!conn NI_2?netconn/- UI_1?connack/null

r/- UI_1? setup/UI_1!setupack UI_1?info_last/UI_1!info.UI_1!callproc NI_2?netalert/UI_1!alert NI_2?netconn/UI_1!conn UI_1?connack/null UI_1?connack/null

r/- UI_1?setup/UI_1!setupack UI_1?info_last/UI_1!info.UI_1!callproc NI_2? netalert/UI_1!alert NI_2? netconn/UI_1!conn T305?timeout/- UI_1?connack/null

r/- UI_1? setup/UI_1!setupack UI_1?info_last/UI_1!info.UI_1!callproc NI_2?netalert/UI_1!alert NI_2?netconn/UI_1!conn T308?timeout/- UI_1?connack/null

r/- UI_1? setup/UI_1!setupack UI_1?info_bad/UI_1!prog.UI_1!disc UI_1?setup_bad/- T305?timeout/UI_1!rel

r/- UI_1?setup/UI_1!setupack UI_1?info_bad/UI_1!prog.UI_1!disc UI_1? setup/- T305?timeout/UI_1!rel

r/- UI_1? setup/UI_1!setupack UI_1?info_bad/UI_1!prog.UI_1!disc UI_1?info/- T305?timeout/UI_1!rel

r/- UI_1?setup/UI_1!setupack UI_1?info_bad/UI_1!prog.UI_1!disc UI_1?info_bad/- T305?timeout/UI_1!rel

r/- UI_1?setup/UI_1!setupack UI_1?info_bad/UI_1!prog.UI_1!disc UI_1?info_last/- T305?timeout/UI_1!rel

r/- UI_1?setup/UI_1!setupack UI_1?info_bad/UI_1!prog.UI_1!disc UI_1?rel/UI_1!relcom UI_1?setup_bad/UI_1!relcom

r/- UI_1? setup/UI_1!setupack UI_1?info_bad/UI_1!prog.UI_1!disc UI_1?disc/UI_1!rel UI_1?disc/null

r/- UI_1? setup/UI_1!setupack UI_1?info_bad/UI_1!prog.UI_1!disc T302?timeout/- T305?timeout/UI_1!rel

r/- UI_1?setup/UI_1!setupack UI_1?info_bad/UI_1!prog.UI_1!disc NI_2?netclear/- T305?timeout/UI_1!rel

r/- UI_1?setup/UI_1!setupack UI_1?info_bad/UI_1!prog.UI_1!disc NI_2? netalert/- T305?timeout/UI_1!rel

r/- UI_1?setup/UI_1!setupack UI_1?info_bad/UI_1!prog.UI_1!disc NI_2? netconn/- T305?timeout/UI_1!rel

r/- UI_1?setup/UI_1!setupack UI_1?info_bad/UI_1!prog.UI_1!disc UI_1?connack/- T305?timeout/UI_1!rel

r/- UI_1?setup/UI_1!setupack UI_1?info_bad/UI_1!prog.UI_1!disc T305?timeout/UI_1!rel UI_1?disc/null

r/- UI_1?setup/UI_1!setupack UI_1?info_bad/UI_1!prog.UI_1!disc T308?timeout/- T305?timeout/UI_1!rel

r/- UI_1?setup/UI_1!setupack UI_1?disc/UI_1!rel UI_1?setup_bad/- UI_1?disc/null

r/- UI_1?setup/UI_1!setupack UI_1?disc/UI_1!rel UI_1?setup/- UI_1?disc/null

r/- UI_1?setup/UI_1!setupack UI_1?disc/UI_1!rel UI_1?info/- UI_1?disc/null

r/- UI_1?setup/UI_1!setupack UI_1?disc/UI_1!rel UI_1?info_bad/- UI_1?disc/null

r/- UI_1?setup/UI_1!setupack UI_1?disc/UI_1!rel UI_1?info_last/- UI_1?disc/null

r/- UI_1?setup/UI_1!setupack UI_1?disc/UI_1!rel UI_1?rel/- UI_1?disc/null

$\mathrm{r} /$ - UI_1?setup/UI_1!setupack UI_1?disc/UI_1!rel UI_1?disc/null UI_1?disc/null

r/- UI_1? setup/UI_1!setupack UI_1?disc/UI_1!rel T302?timeout/- UI_1?disc/null

r/- UI_1? setup/UI_1!setupack UI_1?disc/UI_1!rel NI_2?netclear/- UI_1?disc/null

r/- UI_1?setup/UI_1!setupack UI_1?disc/UI_1!rel NI_2?netalert/- UI_1?disc/null

r/- UI_1?setup/UI_1!setupack UI_1?disc/UI_1!rel NI_2?netconn/- UI_1?disc/null

r/- UI_1?setup/UI_1!setupack UI_1?disc/UI_1!rel UI_1?connack/- UI_1?disc/null

r/- UI_1?setup/UI_1!setupack UI_1?disc/UI_1!rel T305?timeout/- UI_1?disc/null

r/- UI_1?setup/UI_1!setupack UI_1?disc/UI_1!rel T308?timeout/UI_1!rel UI_1?setup_bad/UI_1!relcom 